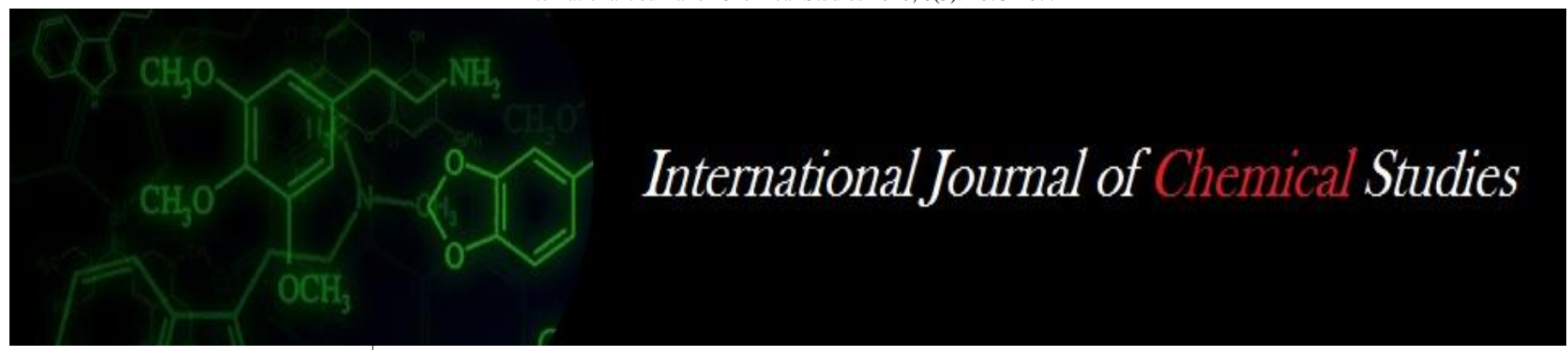

P-ISSN: 2349-8528

E-ISSN: 2321-4902

www.chemijournal.com

IJCS 2020; 8(5): 1073-1077

(C) 2020 IJCS

Received: 25-06-2020

Accepted: 22-08-2020

\section{Kavanashree $\mathbf{K}$}

Department of Plant Pathology,

University of Agricultural

Sciences, Dharwad, Karnataka, India

Shamarao Jahagirdar

Department of Plant Pathology,

University of Agricultural

Sciences, Dharwad, Karnataka,

India

\section{Patil MS}

Department of Plant Pathology,

University of Agricultural

Sciences, Dharwad, Karnataka, India

\section{Kambrekar DN}

Department of Agricultural

Entomology, University of

Agricultural Sciences, Dharwad,

Karnataka, India

Basavaraja GT

AICRP on Soybean, University

of Agricultural Sciences,

Dharwad, Karnataka, India

Krishnaraj PU

Department of Agricultural

Microbiology, University of

Agricultural Sciences, Dharwad,

Karnataka, India

Corresponding Author:

Kavanashree K

Department of Plant Pathology,

University of Agricultural

Sciences, Dharwad, Karnataka,

India

\section{Influence of different nanoformulations on soybean seed quality parameters}

\author{
Kavanashree K, Shamarao Jahagirdar, Patil MS, Kambrekar DN, \\ Basavaraja GT and Krishnaraj PU
}

DOI: https://doi.org/10.22271/chemi.2020.v8.i5o.10439

\begin{abstract}
Application of nanoscience in phytopathology has shown a rapid progress in recent days. Nanoparticles applied through seed treatment improves seed germination, growth and seedling vigour along with its properties of antimicrobial effect. In the present study, influence of four nanoformulations viz. chitosan based zinc, Pseudomonas fluorescens based zinc, pomegranate aril based sulphur and pomegranate aril based silver nanoformulation was assessed on soybean seed quality parameters. All four nanoformulation promoted germination per cent, seedling length, seedling vigour and dry weight. Pomegranate aril based silver nanoformulation @ 500 ppm was found best because, it suppressed fungal infection (97.33\%) during germination effectively in addition to positive influence on seedling growth (seedling vigour index- 2831), which is on par with Carboxin $37.5 \%$ + Thiram $37.5 \%$ DS (2 g/Kg of seed) fungicide. A trend of decreasing germination per cent and seedling vigour index of soybean JS 335 seeds were observed while using higher concentration (above $1000 \mathrm{ppm}$ ) of pomegranate aril based sulphur nanoformulation due to its toxicity. Further, these results can be effeciently utilized in choosing nanoformulation for management of diseases.
\end{abstract}

Keywords: Nanoformulation, seed quality parameters, soybean, fungal infection

\section{Introduction}

Nanotechnology, in recent days has been widely adapted in different fields of science. One such extensively explored field is agriculture. The agricultural sector has set in motion research on this technology to develop agricultural inputs and resources. Nanotechnology studies in phytopathology have developed exponentially over last decade. The topic of nanotechnology deals with synthesis, analysis and manipulation of nano-scale matter within the size range of 1-100 nm, which is called as nanoparticle (Rajan, 2004) ${ }^{[6]}$. Application of nanotechnology in Plant Pathology is a state of art and science that are used for treatment and control of plant diseases and their causal organisms by avoiding disease epidemics.

Soybean (Glycine max (L.)), a legume species is a cheap and rich protein source. It is also an important oilseed crop grown in diverse climatic conditions of India. The main approach for management of different diseases of soybean is use of chemicals. The environmental hazardous nature of chemicals always led to the search of alternative management strategies. Nanofungicide is such one alternative which is a leading strategy in recent days. In addition to diseadse controlling ability, nanofungicides should not have negative impact on growth of seedlings. It is said be best if it positively contributes to the seedling growth. In this regard, synthesized nanoformulation along with the base materials used for synthesis was used in soybean seed treatment to study the impact of the formulation on germination, reduction in fungal infection and seedling vigour. The results are discussed in this paper.

\section{Material and methods}

Chitosan and Pseudomonas fluorescens based zinc nanoparticles synthesized by following procedure of Vinay et al. (2016) ${ }^{[10]}$ and Vinay et al. (2018) ${ }^{[11]}$ respectively along with pomegranate aril based silver and sulphur nanoparticles synthesized by following Srikanth (2018) were tested for its efficacy on germination of JS 335 variety of soybean seed and control of fungal infection. In addition effect of nanoparticles on seedling vigour was assessed. 
The effect of seed treatment with four nanoparticles along with base materials and standard check fungicide on soybean seed germination and seedling vigour index was studied by adopting the procedure of 'Between Paper' (Rolled towel paper) as described by ISTA rules (Anon., 1999) ${ }^{[2]}$. The soybean seeds were soaked in various concentration of different nanoparticle (as detailed in table 1) for 5 minutes and dried on a blotter paper. Randomly selected 400 seeds from the treatment were placed in between four pairs of moist germination paper and rolled with a plastic cover. This was placed in germination chamber at $26{ }^{\circ} \mathrm{C}$ and 95 per cent Relative humidity. Germination percentage, fungal infection, shoot and root length was recorded for three replication of each treatment after 8 days. Seedling vigour index was calculated by the following formula, given by Abdul Baki and Anderson (1973) ${ }^{[1]}$

Seedling Vigour Index $=$ Seed germination $(\%) \times$ Seedling length (Shoot + Root length $(\mathrm{cm})$ )

Further 10 seedlings were taken from each treatment, dried in hot air oven at $75{ }^{\circ} \mathrm{C}$ for 2-3 days and weight was recorded. Three replications were maintained.

\section{Results and discussion}

The seed treatment effect of Chitosan based zinc, Pseudomonas fluorescens based zinc, Pomegranate aril based sulphur and silver nanoformulations on soybean seed quality parameters viz. germination, fungal infection (Table 1), seedling vigour and dry weight (Table 2 ) has discussed below in detail.

\section{Effect of nanoformulations on germination and fungal infection}

Germination of JS 335 seeds of soybean among different tested concentration of nanoformulation varied from 94 to 99.67 per cent. Carboxin $37.5 \%$ + Thiram 37.5\% DS (2 g/Kg of seed), a chemical fungicide resulted in 99.67 per cent germination which was on par with all four nanoformulation and lowest was observed in control. Pomegranate aril based silver nanoformulation at $500 \mathrm{ppm}$ concentration suppressed fungal infection (Aspergillus spp.) effectively to the extent of 97.33 per cent against 77 per cent in control. This treatment is on par with Carboxin $37.5 \%$ + Thiram $37.5 \%$ DS $(2 \mathrm{~g} / \mathrm{Kg}$ of seed) treatment with 98.33 percent reduction. These two treatments significantly differed from all other treatments (Table 1). This result is concurrent with the results of Supriya, 2019 who reported that germination of seeds in different tested nanoparticle did not differ significantly. The research findings of Maity et al. (2016) is also evidenced for increase in germination and reduction in fungal infection using zinc and silver nanoparticles for sorghum and cowpea seed treatments.

\section{Effect of nanoformulations on seedling length, seedling vigour and dry weight}

The seedling length, seedling vigour index and dry weight of soybean seeds treated with nanoformulations are represented in table 2 and plate 1 . Among four tested nanoformulation along with base materials used in synthesis, there was no significant difference in shoot length, but these treatments differed significantly from control treatment with $7.87 \mathrm{~cm}$. However, root length varied significantly among treatments. Maximum root length was observed in the seeds treated with pomegranate aril based silver nanoformulation with $19.57 \mathrm{~cm}$ and this is on par with many treatments as indicated in table 2. The lowest was observed in control with $13.80 \mathrm{~cm}$ and is on par with chitosan based zinc nanoformulation treated with 500 ppm concentration. Seedling length was highest in Carboxin $37.5 \%$ + Thiram $37.5 \%$ DS traetment $(31.03 \mathrm{~cm})$ and the result was on par with all other treatment except control $(21.67 \mathrm{~cm})$. Seedling vigour index, an important seed quality parameter was highest in Carboxin 37.5\% + Thiram 37.5\% DS traetment (3092.99) and this treatment was statistically on par with different treatments. Nanoformulations treatments among them are, chitosan and Pseudomonas fluorescens based zinc nanoformulations at 1000, 1250 and $1500 \mathrm{ppm}$, pomegranate aril based sulphur nanoformulation at 1500 and 2000 ppm, and pomegranate aril based silver nanoformualtion at all four concentration. A lowest seedling vigour index of 2123.33 was recorded in control. Seedling dry weigh varied from $0.64 \mathrm{~g}$ (Pomegranate aril based silver nanoformulation @ $50 \mathrm{ppm}$ ) to $0.87 \mathrm{~g}$ (Pseudomonas fluorescens based zinc nanoformulation @1500 ppm). Similar observations were recorded by Hojjat (2015) ${ }^{[4]}$ and Supriya (2019) ${ }^{[9]}$ where seed treatment with silver nanoparticles resulted in improvement of seed germination per cent, seedling vigour index and dry weight in fenugreek and soybean respectively. The result is also in agreement with the experimental result of Hao et al. (2016) ${ }^{[3]}$ who reported the significant promotion of root and shoot length with the treatment of iron and titanium nanoparticles in rice seeds and also observed non obvious effect on weight. A decrease in seedling vigour index was observed in pomegranate aril based nanoformulation after $1000 \mathrm{ppm}$ concentration. This result was in accordance with the observation found by Salem et al. (2016) ${ }^{[7]}$, who observed an inhibitory effect of suphur nanoparticle on tomato seeds beyond $300 \mathrm{ppm}$ concentration.

Table 1: Effect of seed treatment of nanoformulation on germination per cent and fungal infection rate in soybean

\begin{tabular}{|c|c|c|c|}
\hline Treatments & Concentration & Germination percentage $^{*}(\%)$ & Fungal infection* $(\%)$ \\
\hline \multirow[t]{4}{*}{ Chitosan based zinc oxide nanoformulation } & $500 \mathrm{ppm}$ & $\begin{array}{l}94.67^{\mathrm{de}} \\
(77.09)\end{array}$ & $\begin{array}{c}12.00^{\text {efghi }} \\
(20.26)\end{array}$ \\
\hline & $1000 \mathrm{ppm}$ & $\begin{array}{c}97.00^{\text {abcde }} \\
(80.12)\end{array}$ & $\begin{array}{c}11.33^{\text {efghi }} \\
(19.65)\end{array}$ \\
\hline & $1250 \mathrm{ppm}$ & $\begin{array}{c}98.00^{\text {abcd }} \\
(83.67)\end{array}$ & $\begin{array}{c}9.00^{\mathrm{de}} \\
(17.44)\end{array}$ \\
\hline & $1500 \mathrm{ppm}$ & $\begin{array}{c}98.67^{\text {abcd }} \\
(84.47)\end{array}$ & $\begin{array}{c}6.67^{\text {cd }} \\
(14.85)\end{array}$ \\
\hline \multirow[t]{4}{*}{ Pseudomonas fluorescens based zinc nanoformulation } & $500 \mathrm{ppm}$ & $\begin{array}{l}95.67^{\text {cde }} \\
(78.33)\end{array}$ & $\begin{array}{l}13.00^{\text {fghi }} \\
(21.13)\end{array}$ \\
\hline & $1000 \mathrm{ppm}$ & $\begin{array}{l}96.00^{\text {abcde }} \\
(81.04)\end{array}$ & $\begin{array}{l}10.00^{\text {defgh }} \\
(18.38)\end{array}$ \\
\hline & $1250 \mathrm{ppm}$ & $\begin{array}{c}97.67^{\text {abcde }} \\
(82.88)\end{array}$ & $\begin{array}{c}7.00^{\text {cd }} \\
(14.68)\end{array}$ \\
\hline & $1500 \mathrm{ppm}$ & $97.67^{\text {abcde }}$ & $7.00^{\mathrm{cd}}$ \\
\hline
\end{tabular}




\begin{tabular}{|c|c|c|c|}
\hline & & $(82.88)$ & $(15.26)$ \\
\hline \multirow[t]{4}{*}{ Pomegranate aril based sulphur nanoformulation } & $500 \mathrm{ppm}$ & $\begin{array}{c}98.00^{\text {abcde }} \\
(81.87)\end{array}$ & $\begin{array}{l}14.00^{\text {hi }} \\
(21.97)\end{array}$ \\
\hline & $1000 \mathrm{ppm}$ & $\begin{array}{c}99.33^{\mathrm{abc}} \\
(86.07)\end{array}$ & $\begin{array}{c}14.67^{\mathrm{i}} \\
(22.49)\end{array}$ \\
\hline & $1500 \mathrm{ppm}$ & $\begin{array}{c}97.00^{\text {abcde }} \\
(80.12)\end{array}$ & $\begin{array}{l}9.33^{\text {defg }} \\
(17.69)\end{array}$ \\
\hline & 2000 ppm & $\begin{array}{c}93.00^{\mathrm{e}} \\
(74.76)\end{array}$ & $\begin{array}{c}10.00^{\text {defgh }} \\
(18.38)\end{array}$ \\
\hline \multirow[t]{4}{*}{ Pomegranate aril based silver nanoformulation } & $50 \mathrm{ppm}$ & $\begin{array}{c}96.33^{\text {bcde }} \\
(79.14)\end{array}$ & $\begin{array}{c}8.33^{\mathrm{de}} \\
(16.69)\end{array}$ \\
\hline & $100 \mathrm{ppm}$ & $\begin{array}{c}96.67^{\text {abcde }} \\
(79.66)\end{array}$ & $\begin{array}{l}9.33^{\text {defg }} \\
(17.78)\end{array}$ \\
\hline & $250 \mathrm{ppm}$ & $\begin{array}{c}98.00^{\mathrm{abcd}} \\
(83.67)\end{array}$ & $\begin{array}{c}5.00^{\mathrm{bc}} \\
(12.88)\end{array}$ \\
\hline & 500 ppm & $\begin{array}{l}99.33^{\mathrm{ab}} \\
(87.07)\end{array}$ & $\begin{array}{c}2.67^{\mathrm{a}} \\
(9.36)\end{array}$ \\
\hline Bulk Zinc oxide & $1250 \mathrm{ppm}$ & $\begin{array}{c}96.33^{\text {bcde }} \\
(79.24)\end{array}$ & $\begin{array}{c}6.67^{\mathrm{cd}} \\
(14.90)\end{array}$ \\
\hline Sodium thiosulphate & $2000 \mathrm{ppm}$ & $\begin{array}{l}95.00^{\mathrm{de}} \\
(77.12)\end{array}$ & $\begin{array}{l}13.67^{\mathrm{hi}} \\
(21.68)\end{array}$ \\
\hline Silver nitrate & $500 \mathrm{ppm}$ & $\begin{array}{c}98.00^{\text {abcd }} \\
(83.24)\end{array}$ & $\begin{array}{c}6.67^{\mathrm{cd}} \\
(14.72)\end{array}$ \\
\hline Water soluble chitosan & $1 \%$ & $\begin{array}{c}96.33 b^{\text {cde }} \\
(79.14)\end{array}$ & $\begin{array}{l}13.33^{\mathrm{ghi}} \\
(21.40)\end{array}$ \\
\hline Pseudomonas fluorescens extract & $20 \%$ & $\begin{array}{c}95.67^{\text {cde }} \\
(78.26)\end{array}$ & $\begin{array}{l}9.33^{\text {defg }} \\
(17.78)\end{array}$ \\
\hline Pomegranate aril extract & $10 \%$ & $\begin{array}{c}97.33^{\text {abcde }} \\
(80.92)\end{array}$ & $\begin{array}{c}10.00^{\text {defgh }} \\
(18.30)\end{array}$ \\
\hline Carbendazim 50\% WP & $2 \mathrm{~g} / \mathrm{Kg}$ & $\begin{array}{c}96.33^{\text {bcde }} \\
(79.14)\end{array}$ & $\begin{array}{c}8.67^{\mathrm{de}} \\
(17.08)\end{array}$ \\
\hline Carboxin $37.5 \%+$ Thiram $37.5 \%$ DS & $2 \mathrm{~g} / \mathrm{Kg}$ & $\begin{array}{c}99.67^{\mathrm{a}} \\
(87.87)\end{array}$ & $\begin{array}{l}1.67^{a} \\
(615)\end{array}$ \\
\hline Control & & $\begin{array}{l}94.00^{\mathrm{de}} \\
(76.09)\end{array}$ & $\begin{array}{c}23.00^{\mathrm{j}} \\
(28.61)\end{array}$ \\
\hline S.Em. \pm & & 2.43 & 1.34 \\
\hline C.D. $(0.01)$ & & 9.21 & 5.08 \\
\hline C.V. $(\%)$ & & 5.20 & 13.21 \\
\hline
\end{tabular}

*Values are mean of three replication

Figures in parenthesis represents arc sine transformed value

Table 2: Influence of nanoformulations on seedling growth, seedling vigour index and seedling dry weigh

\begin{tabular}{|c|c|c|c|c|c|c|}
\hline Treatments & Concentration & $\begin{array}{l}\text { Shoot length* } \\
(\mathrm{cm})\end{array}$ & $\begin{array}{l}\text { Root length* } \\
(\mathrm{cm})\end{array}$ & $\begin{array}{c}\text { Seedling length* } \\
(\mathrm{cm})\end{array}$ & $\begin{array}{c}\text { Seedling } \\
\text { vigour }\end{array}$ & $\begin{array}{c}\text { Dry weight }{ }^{*} \\
\text { (g) }\end{array}$ \\
\hline \multirow{4}{*}{ Chitosan based zinc oxide nanoformulation } & $500 \mathrm{ppm}$ & $\begin{array}{l}12.68^{a} \\
(3.56)\end{array}$ & $\begin{array}{c}14.05^{\mathrm{de}} \\
(3.75)\end{array}$ & $\begin{array}{l}26.73^{\mathrm{a}} \\
(5.17)\end{array}$ & $\begin{array}{c}2566.40^{\mathrm{bc}} \\
(50.64)\end{array}$ & $\begin{array}{c}0.80^{\text {bcd }} \\
(0.89)\end{array}$ \\
\hline & $100 \mathrm{ppm}$ & $\begin{array}{l}12.82^{\mathrm{a}} \\
(3.58)\end{array}$ & $\begin{array}{c}16.67^{\text {abcde }} \\
(4.08)\end{array}$ & $\begin{array}{l}29.48^{a} \\
(5.43)\end{array}$ & $\begin{array}{c}2928.68^{\mathrm{ab}} \\
(54.08)\end{array}$ & $\begin{array}{l}0.80^{\mathrm{bcd}} \\
(0.89)\end{array}$ \\
\hline & $1250 \mathrm{ppm}$ & $\begin{array}{l}12.93^{\mathrm{a}} \\
(3.60)\end{array}$ & $\begin{array}{c}15.45^{\text {cde }} \\
(3.93)\end{array}$ & $\begin{array}{l}30.18^{\mathrm{a}} \\
(5.35) \\
\end{array}$ & $\begin{array}{c}2957.64^{\mathrm{ab}} \\
(52.95) \\
\end{array}$ & $\begin{array}{l}0.85^{\mathrm{ab}} \\
(0.92) \\
\end{array}$ \\
\hline & $1500 \mathrm{ppm}$ & $\begin{array}{l}11.66^{\mathrm{a}} \\
(3.41)\end{array}$ & $\begin{array}{c}14.87^{\text {cde }} \\
(3.85)\end{array}$ & $\begin{array}{l}29.00^{\mathrm{a}} \\
(5.46)\end{array}$ & $\begin{array}{c}2813.00^{\mathrm{abc}} \\
(54.26)\end{array}$ & $\begin{array}{l}0.83^{\mathrm{abc}} \\
(0.91)\end{array}$ \\
\hline \multirow{4}{*}{$\begin{array}{c}\text { Pseudomonas fluorescens based zinc } \\
\text { nanoformulation }\end{array}$} & 500 ppm & $\begin{array}{l}12.30^{\mathrm{a}} \\
(3.51)\end{array}$ & $\begin{array}{l}14.87^{\text {cde }} \\
(3.86)\end{array}$ & $\begin{array}{l}27.17^{\mathrm{a}} \\
(5.21)\end{array}$ & $\begin{array}{l}2598.94^{\mathrm{bc}} \\
(50.97)\end{array}$ & $\begin{array}{l}0.83^{\mathrm{abc}} \\
(0.91)\end{array}$ \\
\hline & $1000 \mathrm{ppm}$ & $\begin{array}{l}11.97^{\mathrm{a}} \\
(3.44)\end{array}$ & $\begin{array}{l}17.38^{\mathrm{abc}} \\
(4.16)\end{array}$ & $\begin{array}{l}29.35^{\mathrm{a}} \\
(5.40)\end{array}$ & $\begin{array}{c}2876.30^{\mathrm{abc}} \\
(53.45)\end{array}$ & $\begin{array}{l}0.84^{\mathrm{ab}} \\
(0.91) \\
\end{array}$ \\
\hline & 1250 ppm & $\begin{array}{l}12.18^{\mathrm{a}} \\
(3.49)\end{array}$ & $\begin{array}{l}15.48^{\text {cde }} \\
(3.92)\end{array}$ & $\begin{array}{l}27.66^{\mathrm{a}} \\
(5.25)\end{array}$ & $\begin{array}{l}2701.46^{\mathrm{abc}} \\
(51.92)\end{array}$ & $\begin{array}{l}0.84^{\mathrm{ab}} \\
(0.92)\end{array}$ \\
\hline & 1500 ppm & $\begin{array}{l}12.60^{\mathrm{a}} \\
(3.55)\end{array}$ & $\begin{array}{c}17.50^{\mathrm{abc}} \\
(4.18)\end{array}$ & $\begin{array}{l}30.10^{\mathrm{a}} \\
(5.49)\end{array}$ & $\begin{array}{l}2939.77^{\mathrm{ab}} \\
(54.21)\end{array}$ & $\begin{array}{l}0.87^{\mathrm{a}} \\
(0.93)\end{array}$ \\
\hline \multirow{4}{*}{$\begin{array}{l}\text { Pomegranate aril based sulphur } \\
\text { nanoformulation }\end{array}$} & $500 \mathrm{ppm}$ & $\begin{array}{l}13.13^{\mathrm{a}} \\
(3.62)\end{array}$ & $\begin{array}{c}16.40^{\text {abcde }} \\
(4.05)\end{array}$ & $\begin{array}{l}29.53^{\mathrm{a}} \\
(5.43)\end{array}$ & $\begin{array}{c}2864.73^{\mathrm{abc}} \\
(53.51)\end{array}$ & $\begin{array}{l}0.66^{\mathrm{gh}} \\
(0.81)\end{array}$ \\
\hline & $1000 \mathrm{ppm}$ & $\begin{array}{l}11.52^{\mathrm{a}} \\
(3.38)\end{array}$ & $\begin{array}{c}17.50^{\mathrm{abc}} \\
(4.18)\end{array}$ & $\begin{array}{l}29.02^{\mathrm{a}} \\
(5.38)\end{array}$ & $\begin{array}{c}2698.55^{\mathrm{abc}} \\
(51.92)\end{array}$ & $\begin{array}{l}0.73^{\text {def }} \\
(0.85)\end{array}$ \\
\hline & $1500 \mathrm{ppm}$ & $\begin{array}{l}12.73^{\mathrm{a}} \\
(3.57)\end{array}$ & $\begin{array}{l}13.83^{\mathrm{e}} \\
(3.72)\end{array}$ & $\begin{array}{l}26.57^{\mathrm{a}} \\
(5.15)\end{array}$ & $\begin{array}{c}2514.98^{c} \\
(50.15)\end{array}$ & $\begin{array}{l}0.76^{\text {cdef }} \\
(0.87)\end{array}$ \\
\hline & 2000 ppm & $\begin{array}{l}13.67^{\mathrm{a}} \\
(3.69)\end{array}$ & $\begin{array}{l}16.52^{\text {abcde }} \\
(4.06)\end{array}$ & $\begin{array}{l}26.53^{\mathrm{a}} \\
(5.15)\end{array}$ & $\begin{array}{c}2617.63^{b c} \\
(50.70)\end{array}$ & $\begin{array}{l}0.70^{\text {fgh }} \\
(0.84)\end{array}$ \\
\hline $\begin{array}{l}\text { Pomegranate aril based silver } \\
\text { nanoformulation }\end{array}$ & 50 ppm & $\begin{array}{l}12.83^{\mathrm{a}} \\
(3.58)\end{array}$ & $\begin{array}{l}16.40^{\text {bcde }} \\
(4.03)\end{array}$ & $\begin{array}{l}29.23^{\mathrm{a}} \\
(5.40)\end{array}$ & $\begin{array}{l}2816.14^{a b c} \\
(52.97)\end{array}$ & $\begin{array}{l}0.64^{\mathrm{h}} \\
(0.80)\end{array}$ \\
\hline
\end{tabular}




\begin{tabular}{|c|c|c|c|c|c|c|}
\hline & $100 \mathrm{ppm}$ & $\begin{array}{l}13.17^{\mathrm{a}} \\
(3.63)\end{array}$ & $\begin{array}{c}15.63^{\text {cde }} \\
(3.95)\end{array}$ & $\begin{array}{l}28.80^{\mathrm{a}} \\
(5.37)\end{array}$ & $\begin{array}{c}2784.00^{\mathrm{abc}} \\
(52.75)\end{array}$ & $\begin{array}{l}0.74^{\mathrm{def}} \\
(0.86)\end{array}$ \\
\hline & $250 \mathrm{ppm}$ & $\begin{array}{l}12.37^{\mathrm{a}} \\
(3.52)\end{array}$ & $\begin{array}{c}16.77^{\text {abcde }} \\
(4.09)\end{array}$ & $\begin{array}{l}28.90^{\mathrm{a}} \\
(5.40)\end{array}$ & $\begin{array}{c}2832.20^{\text {abc }} \\
(53.42)\end{array}$ & $\begin{array}{l}0.74^{\text {def }} \\
(0.86)\end{array}$ \\
\hline & $500 \mathrm{ppm}$ & $\begin{array}{l}13.53^{\mathrm{a}} \\
(3.68)\end{array}$ & $\begin{array}{l}19.57^{\mathrm{a}} \\
(4.42)\end{array}$ & $\begin{array}{l}28.50^{\mathrm{a}} \\
(5.40)\end{array}$ & $\begin{array}{c}2831.00^{\mathrm{abc}} \\
(53.79)\end{array}$ & $\begin{array}{l}0.83^{\text {abc }} \\
(0.91)\end{array}$ \\
\hline Bulk Zinc oxide & $1250 \mathrm{ppm}$ & $\begin{array}{l}13.43^{\mathrm{a}} \\
(3.66)\end{array}$ & $\begin{array}{c}17.07^{\text {abcd }} \\
(4.13)\end{array}$ & $\begin{array}{l}30.50^{\mathrm{a}} \\
(5.52)\end{array}$ & $\begin{array}{c}2938.17^{\mathrm{ab}} \\
(54.16)\end{array}$ & $\begin{array}{l}0.75^{\text {def }} \\
(0.87)\end{array}$ \\
\hline Sodium thiosulphate & $2000 \mathrm{ppm}$ & $\begin{array}{l}11.37^{\mathrm{a}} \\
(3.37)\end{array}$ & $\begin{array}{c}16.67^{\text {abcde }} \\
(4.08)\end{array}$ & $\begin{array}{l}28.03^{\mathrm{a}} \\
(5.29)\end{array}$ & $\begin{array}{c}2635.13^{\text {abc }} \\
(51.33)\end{array}$ & $\begin{array}{l}0.66^{\mathrm{gh}} \\
(0.81)\end{array}$ \\
\hline Silver nitrate & $500 \mathrm{ppm}$ & $\begin{array}{l}12.00^{\mathrm{a}} \\
(3.46)\end{array}$ & $\begin{array}{c}16.93^{\mathrm{abcd}} \\
(4.11)\end{array}$ & $\begin{array}{l}28.93^{\mathrm{a}} \\
(5.37)\end{array}$ & $\begin{array}{c}2748.67^{\mathrm{bc}} \\
(52.38)\end{array}$ & $\begin{array}{l}0.71^{\text {fgh }} \\
(0.84)\end{array}$ \\
\hline Water soluble chitosan & $1 \%$ & $\begin{array}{l}12.83^{\mathrm{a}} \\
(3.58)\end{array}$ & $\begin{array}{c}16.47^{\text {abcde }} \\
(4.06)\end{array}$ & $\begin{array}{l}29.30^{\mathrm{a}} \\
(5.41)\end{array}$ & $\begin{array}{c}2822.57^{\mathrm{bc}} \\
(53.12)\end{array}$ & $\begin{array}{l}0.75^{\text {def }} \\
(0.87)\end{array}$ \\
\hline Pseudomonas fluorescens extract & $20 \%$ & $\begin{array}{l}11.87^{\mathrm{a}} \\
(3.44)\end{array}$ & $\begin{array}{c}15.23^{\text {cde }} \\
(3.90)\end{array}$ & $\begin{array}{l}27.11^{\mathrm{a}} \\
(5.20)\end{array}$ & $\begin{array}{c}2593.20^{\mathrm{bc}} \\
(50.85)\end{array}$ & $\begin{array}{l}0.78^{\text {def }} \\
(0.88) \\
\end{array}$ \\
\hline Pomegranate aril extract & $10 \%$ & $\begin{array}{l}12.70^{\mathrm{a}} \\
(3.56)\end{array}$ & $\begin{array}{c}17.10^{\mathrm{abcd}} \\
(4.13)\end{array}$ & $\begin{array}{l}29.80^{\mathrm{a}} \\
(5.46)\end{array}$ & $\begin{array}{c}2900.53^{\mathrm{ab}} \\
(53.83)\end{array}$ & $\begin{array}{l}0.72^{\text {efg }} \\
(0.85)\end{array}$ \\
\hline Carbendazim & $2 \mathrm{~g} / \mathrm{Kg}$ & $\begin{array}{l}11.40^{\mathrm{a}} \\
(3.38)\end{array}$ & $\begin{array}{c}15.73^{\text {bcde }} \\
(3.97)\end{array}$ & $\begin{array}{l}27.13^{\mathrm{a}} \\
(5.21)\end{array}$ & $\begin{array}{c}2613.84^{\mathrm{bc}} \\
(51.12)\end{array}$ & $\begin{array}{l}0.70^{\text {fgh }} \\
(0.84)\end{array}$ \\
\hline Carboxin $37.5 \%$ + Thiram $37.5 \%$ DS & $2 \mathrm{~g} / \mathrm{Kg}$ & $\begin{array}{l}12.40^{\mathrm{a}} \\
(3.52)\end{array}$ & $\begin{array}{c}18.97^{\mathrm{ab}} \\
(4.35)\end{array}$ & $\begin{array}{l}31.03^{\mathrm{a}} \\
(5.57)\end{array}$ & $\begin{array}{c}3092.99^{\mathrm{a}} \\
(55.61)\end{array}$ & $\begin{array}{l}0.80^{\text {bcd }} \\
(0.89)\end{array}$ \\
\hline Control & & $\begin{array}{l}7.87^{b} \\
(2.79)\end{array}$ & $\begin{array}{l}13.80^{\mathrm{e}} \\
(3.71)\end{array}$ & $\begin{array}{l}21.67^{b} \\
(4.65)\end{array}$ & $\begin{array}{c}2123.33^{c} \\
(46.05)\end{array}$ & $\begin{array}{l}0.66^{\mathrm{gh}} \\
(0.81)\end{array}$ \\
\hline S.Em. \pm & & 0.11 & 0.11 & 0.13 & 1.32 & 0.01 \\
\hline C.D. $(0.01)$ & & 0.42 & 0.43 & 0.51 & 4.98 & 0.05 \\
\hline C.V. $(\%)$ & & 5.51 & 4.87 & 4.34 & 4.34 & 2.82 \\
\hline
\end{tabular}

*Values are mean of three replication

Figures in parenthesis are square root transformed value

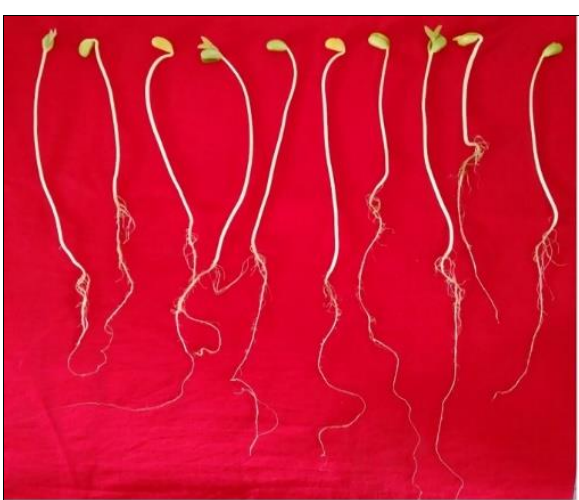

Chitosan based zinc nanoformulation

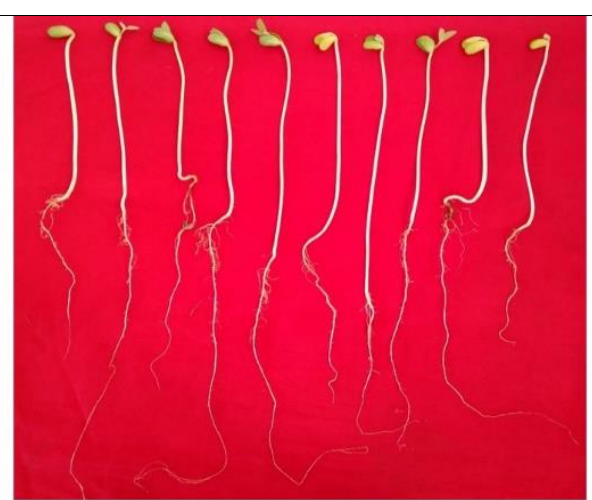

Pseudomonas fluorescens based zinc nanoformulation

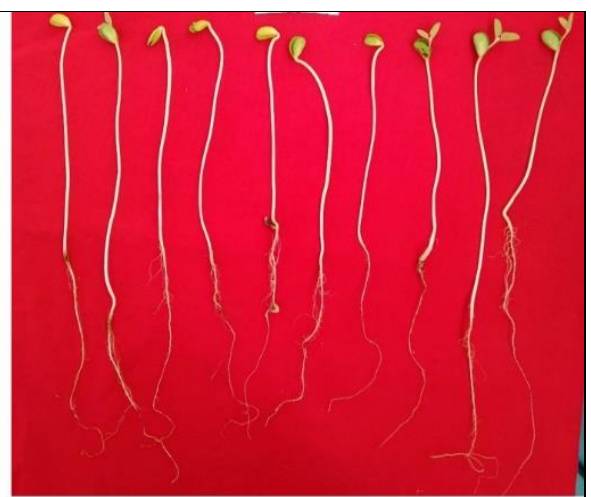

Pomegranate aril based sulphur nanoformulation

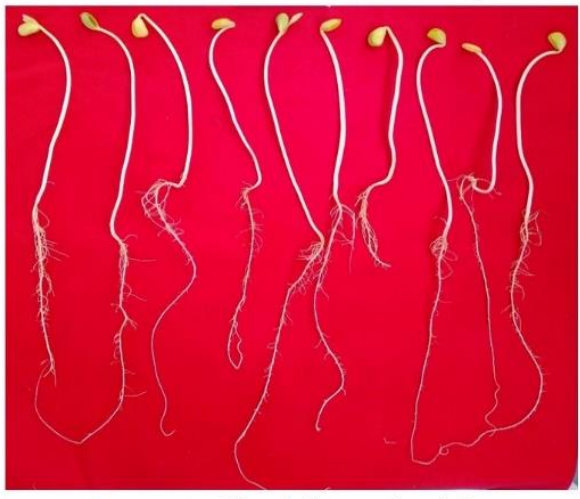

Pomegranate aril based silver nanoformulation

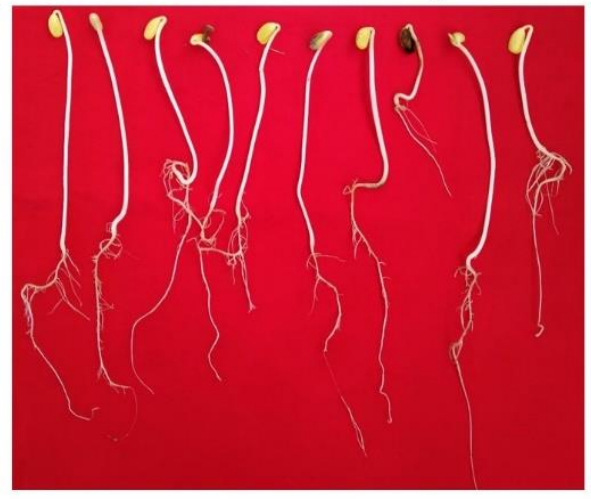

Water contro]

Plate 1: Effect of different nanoformulation on seedling growth

\section{Conclusion}

Nanotechnology application in phytopathology has been widely spreading globally. In this view, an attempt was made to analyze the effect of chitosan and Pseudomonas based zinc nanoformulation and pomegranate aril based sulphur and silver nanoformulation on soybean seed quality parameters. All four nanoformulation, promoted germination shoot length, root length, seedlng vigour. Pomegranate aril based silver nanoformulation, not only showed positive effect on seedling vigour, but also suppressed fungal pathogens effectively. Thus, this teatment was found to be best. These nanoformulations can be further effectively used for management of various phytopathogens as they promote and strengthen the seedling in addition to pathogen suppression. 


\section{References}

1. Abdul Baki AA, Anderson JO. Vigour determination in soybean seed by multiple criteria. Crop Sci. 1973; 13:630-632.

2. Anonymous. International Rules of Seed Testing. Seed Sci. and Technol. 1999; 4:180.

3. Hao Y, Zhang Z, Rui Y, Ren J, Hou T, Wu S et al. Effect of different nanoparticles on seed germination and seedling growth in rice. $2^{\text {nd }}$ Annual International Conference on Advanced Material Engineering. 2016, 166-173.

4. Hojjat SS. Impact of silver nanoparticles on germinated fenugreek seed. Int. J Agri. Crop Sci. 2015; 8:627-630.

5. Maity A, Natarajan N, Pastor M, Vijay D, Gupta CK, Wasnik VK. Nanoparticle influence seed germination traits and seed pathogen infection rate in forage sorghum (Sorghum bicolour) and cowpea (Vigna unguiculata). Indian J Exp. Biol. 2018; 56:363-372.

6. Rajan MS Nano. The Next Revolution, National Book Trust, New Delhi, India. 2014, 15-72.

7. Salem NM, Albanna LS, Awwad AM, Ibrahim QM, Abdeen AO. Sulfur nanoparticles improves root and shoot growth of tomato. J Agric. Sci. 2016; 8(4):179:185.

8. Srikanth HN. Synthesis, characterization and evaluation of green sulphur and silver nanoparticles against foliar fungal pathogens of greengram, M.Sc thesis, UAS, Dharwad. 2018.

9. Supriya ML. Studies on perpetuation of Phakopsora pachyrhizi Syd. causing soybean rust and its management through nanoformulations. M. Sc. thesis, University of Agricultural Sciemces, Dharwad, 2019.

10. Vinay JU, Nargund VB, Jahagirdar S, Hegde RV, Patil RR, Chikkannaswamy. Synthesis and characterization of chitosan based zinc nanoparticles. In: National Symosium on Recent Advances in Plant Health Management for Sustainable Productivity, University of Agricultural Sciences, Dharwad. 2016, 15-16.

11. Vinay JU, Nargund VB, Jahagirdar S, Patil RR, Hegde RV. Green synthesis of zinc nanoparticles using Pseudomonas fluorescens extract and their antibacterial activity against Xanthomonas spp. Int. J Curr. Microbiol. App. Sci. 2018; 7(10):1280-1291. 